\title{
Evaluation of Spontaneous Swallow Frequency in Healthy People and Those With, or at Risk of Developing, Dysphagia: A Systematic Review and Meta-Analysis.
}

Joseph M Bulmer ( $\boldsymbol{\nabla}$ joseph.bulmer@nhs.net )

Newcastle Upon Tyne Hospitals NHS Trust: Newcastle Upon Tyne Hospitals NHS Foundation Trust

https://orcid.org/0000-0001-7778-0840

\section{Caroline Ewers}

City Hospitals Sunderland NHS Foundation Trust: South Tyneside and Sunderland NHS Foundation Trust

\section{Michael J Drinnan}

Newcastle Upon Tyne Hospitals NHS Trust: Newcastle Upon Tyne Hospitals NHS Foundation Trust

\section{Victoria C Ewan}

South Tees Hospitals NHS Trust: South Tees Hospitals NHS Foundation Trust

\section{Research article}

Keywords: swallow frequency, deglutition, deglutition disorders, dysphagia, screening, swallowing problems

Posted Date: January 28th, 2021

DOI: https://doi.org/10.21203/rs.3.rs-153473/v1

License: (9) (1) This work is licensed under a Creative Commons Attribution 4.0 International License. Read Full License 


\section{Abstract}

Background

Dysphagia is a common, and frequently undetected, complication of many neurological disorders and of sarcopoenia in ageing persons. However it is difficult to detect dysphagia clinically until the point of visible aspiration, and there are relatively few trained speech and language therapists, whose time and remit are limited to those with obvious disorders. Reduction in spontaneous swallowing frequency (SSF) has been mooted as a possible proxy for dysphagia. We therefore conducted a systematic review of the literature to describe SSF in both the healthy population, and in disease specific populations, in order to assess its utility as a screening tool to identify dysphagia.

Methods

We searched Medline, Embase, Cochrane Database of Systematic Reviews, and Cochrane Central Register of Controlled Trials databases. Metadata were extracted, collated, and analysed via a random effects model to give quantitative insight.

Results

Three hundred and twelve articles were retrieved, with 19 meeting inclusion and quality criteria. Heterogeneity between studies was high $\left(I^{2}=98 \%\right)$, in part, due to the variety of methods for swallow identification reported. The mean SSF in Healthy younger groups was $0.98 / \mathrm{min}$ [Cl: $0.78 ; 1.23$ ]. In the Parkinson's groups mean SSF was $0.59 / \mathrm{min}[0.41 ; 0.85]$. Mean SSF in Healthy older, Higher risk and Dysphagic populations were similar $(0.21 / \mathrm{min}$ $[0.12 ; 0.37], 0.26 / \mathrm{min}[0.14 ; 0.85]),(0.27 / \mathrm{min}[0.17 ; 0.43]$ respectively).

\section{Conclusions}

SSF is a novel, non-invasive clinical variable which warrants further explorations as to its potential to identify persons at risk of dysphagia. Larger, well-conducted studies are needed to develop objective, standardised methods for detecting SSF, and develop normative values in healthy populations.

\section{Background}

Spontaneous swallowing is one of a group of aerodigestive reflexes supporting airway protection [1]. Swallowing impairment, also known as dysphagia, is a common, and frequently undetected, complication of many neurological and structural disorders in children and adults. Misdirection of oral material into the airway is known as aspiration, and is a common consequence of dysphagia. Dysphagia and aspiration are the pre-eminent risk factors for pneumonia, each with odds ratios $>10[2,3]$.

With 479,564 finished consultant episodes and $>3$ million bed days, pneumonia is the most common cause for UK hospital admissions (HES data: 2015-16), and now the commonest healthcare-associated infection (HCAl) in Europe, accounting for $26 \%$ of all HCAl [4]. But while precautionary assessment by a speech \& language therapist (SALT) would seem prudent, widespread screening of at-risk populations such as stroke patients and the elderly is logistically impossible; only patients of particular concern have a detailed swallow assessment. 
A number of swallow screening tools and techniques may have a place in dysphagia screening; these include questionnaires, observations and clinical history. However, outside of the stroke population, there is no consensus on best practice; each professional chooses their preferred technique [5]. Questionnaires are an effective way to screen community and secondary care patients [6] but remain subjective with varying degrees of diagnostic accuracy due largely to the involuntary nature of the patients' condition. Importantly, there is currently no validated bedside screening tool for objective automated screening of dysphagia and aspiration risk outside of stroke care. Clinical assessment and research in hospital-acquired and aspiration pneumonias are hampered by an inconsistent approach to measuring dysphagia.

We and others are assessing the utility of spontaneous swallowing frequency (SSF) [7] in this role. SSF is the rate of swallowing over a prolonged period without purposeful intervention; reduced SSF is mooted as an indicator of dysphagia in clinical populations that include: post-stroke [7]; head and neck cancer [24]; Parkinson's disease [26]; and the elderly $[13,14,15]$. Recent advances in technology have enabled an assortment of cheap, non-invasive swallow detectors. Surface EMG, sound analysis and other techniques have the potential to offer a low-cost, simple, acceptable screening test that might be deployed with only limited training across these and other vulnerable clinical populations, such as children.

Yet since swallowing is one of the most common and readily observed of all physiological processes, it seems remarkable that there is no consensus as to what constitutes a normal SSF, even in a healthy adult population. To illustrate the scale of uncertainty, we noted early research conducted by Lear et al. [8] suggesting that humans swallow between 203-1008 times per day (0.14-0.7/min), with a mean swallow frequency of 585 per day. Whereas recently, Rudney et al. [9] reported that a healthy human will swallow spontaneously between 18 and 400 times an hour (0.3-6.7/ $\mathrm{min})$. These two normal ranges for SSF cover two orders of magnitude, and are barely compatible with one another. A cursory review of the literature suggests further variability with age, aetiologies and comorbidities. In the context of developing a dysphagia screening test, there is no scope to move forward without a better understanding of this aspect of basic swallow physiology.

In this review we assess the literature relating to the identification of spontaneous swallow rates in healthy populations and in clinical populations with, or at risk of developing, dysphagia. Specifically, we wish to:

- Report the spontaneous swallowing frequency in normal and clinically relevant groups.

- Advise on the utility of SSF as a screening tool to identify risk of dysphagia.

\section{Methods}

Details of the protocol for this systematic review were registered on PROSPERO and can be accessed at www.crd.york.ac.uk/PROSPERO/display_record.asp?ID= CRD42019134061.

\section{Search strategy and selection criteria}

We conducted electronic searches to identify relevant primary research articles using the following databases: MEDLINE, Embase, Cochrane Database of Systematic Reviews, and Cochrane Central Register of Controlled Trials. See Table 1 for the full search strategy. 


\begin{tabular}{|c|c|}
\hline Database & Search Strategy \\
\hline Ovid MEDLINE (R) & 1. (swallow* adj2 frequency).mp. \\
\hline \multirow[t]{9}{*}{$1946-2019$} & 2. (swallow* adj2 rate). $\mathrm{mp}$. \\
\hline & 3. spontaneous swallow*.mp. \\
\hline & 4. swallow* per.mp. \\
\hline & 5.1 or 2 or 3 or 4 \\
\hline & 6. Deglutition Disorders/ \\
\hline & 7. Pneumonia, Aspiration/ or Respiratory Aspiration/ \\
\hline & 8. Dysphagia.mp. \\
\hline & 9.6 or 7 or 8 \\
\hline & 10.5 and 9 \\
\hline EMBASE & 1. (swallow* adj2 frequency).mp. \\
\hline \multirow[t]{9}{*}{$1980-2019$} & 2. (swallow* adj2 rate).mp. \\
\hline & 3. spontaneous swallow*.mp. \\
\hline & 4. swallow* per.mp. \\
\hline & 5.1 or 2 or 3 or 4 \\
\hline & 6. Deglutition Disorders/ \\
\hline & 7. Pneumonia, Aspiration/ or Respiratory Aspiration/ \\
\hline & 8. Dysphagia.mp. \\
\hline & 9.6 or 7 or 8 \\
\hline & 10.5 and 9 \\
\hline
\end{tabular}

\section{Table 1. Electronic search strategies to identify primary research articles on spontaneous swallow frequency}

Citations were excluded by a single rater if they: had no abstract; included no human data; or made no reference to spontaneous swallow rate. Abstracts and where appropriate full articles were then reviewed in more detail. Papers were excluded if they: presented only an abstract and not a full paper; included data only from sleeping subjects (i.e. no awake control); did not measure SSF directly (i.e. SSF was predicted using other variables); and if they only reported data from populations under the age of 18 years.

\section{Data extraction}

Where articles met the inclusion criteria, a single rater extracted the following data: sample size; study population/aetiology; study controls; population ages; swallow frequency; standard deviation of swallow 
frequency between subjects; swallow identification method; length of swallowing recordings; statistical significance of SSF between observational and/or intervention groups; and additional information where appropriate - for example, correlational statistics and study information.

\section{Quality assessment}

A full assessment of the quality of the papers was conducted using the NHLBI Study Quality Assessment tools [10]. Two independent raters scored each paper and rated them of poor, fair or good quality (see Appendix A).

\section{Data analysis}

We conducted meta-analysis for the whole group, and for sub-groups of subjects as follows:

Healthy younger below 60 years of age;

Healthy older of 60 years or more;

Dysphagic, with documented dysphagia;

Parkinson's, with a diagnosis of Parkinson's disease;

Higher risk, with aetiologies associated in the literature with dysphagia that do not fit into the other categories e.g. post stroke patients without documented dysphagia.

In each analysis, the outcome measure was the log-transform of the mean spontaneous swallow frequency (SSF) expressed in units of swallows per minute.

\section{Assessment of study heterogeneity}

Heterogeneity is a measure of inconsistency between the groups and studies making up a meta-analysis. High heterogeneity means that the groups are so different that they cannot be considered as samples from the same population. In this case, a random-effects meta-analysis is indicated and the results must be treated with caution; a pooled estimate of the outcome measure may not be meaningful. Heterogeneity was assessed for all groups separately, and for the whole group.

\section{Estimate of population spontaneous swallow frequency}

We estimated the central value and confidence intervals for SSF using a random effects model and inverse variance weighting to pool the studies. We report for all groups separately and for the whole group.

The Meta and Metafor packages from the R programming language [11] were used for data analysis.

\section{Results}

\section{Literature retrieval}


From 312 abstracts found by our search strategy, 19 full papers were included in this review (Figure 1). They are summarised in Tables 2 and 3. 


\begin{tabular}{|c|c|c|c|c|c|c|}
\hline $\begin{array}{l}\text { Author, } \\
\text { year }\end{array}$ & Aetiology & $n$ & $\begin{array}{l}\text { Swallow } \\
\text { rate }\end{array}$ & $P$ & Additional details & $\begin{array}{l}\text { Swallow } \\
\text { identification } \\
\text { method }\end{array}$ \\
\hline $\begin{array}{l}\text { Crary et al. } \\
2014 \\
{[7]}\end{array}$ & $\begin{array}{l}\text { Stroke } \\
\text { patients }\end{array}$ & $\begin{array}{l}26 \\
36\end{array}$ & $\begin{array}{l}\text { Dys: } 0.23 \\
( \pm 0.15) \\
\text { sw/min } \\
\text { Non-dys: } \\
0.55( \pm 0.3) \\
\text { sw/min }\end{array}$ & $<0.0001$ & $\begin{array}{l}\text { Acute stroke patients } \\
\text { screened using SSF } \\
\text { (via acoustic analysis) } \\
\text { vs. standard clinical } \\
\text { screening protocols for } \\
\text { dysphagia } \\
\text { identification. }\end{array}$ & $\begin{array}{l}\text { Microphone } \\
\text { taped lateral } \\
\text { inferior to the } \\
\text { cricoid and } \\
\text { connected to a } \\
\text { digital recorder } \\
\text { [14]. }\end{array}$ \\
\hline $\begin{array}{l}\text { Niimi et al. } \\
2018 \\
{[25]}\end{array}$ & $\begin{array}{l}\text { Stroke } \\
\text { patients }\end{array}$ & $\begin{array}{l}17 \\
23\end{array}$ & $\begin{array}{l}\text { High SP: } \\
0.51 \pm 0.34 \\
\text { Low SP: } \\
0.27 \pm 0.19\end{array}$ & 0.016 & $\begin{array}{l}\text { To determine the } \\
\text { relationship between } \\
\text { SSF and salivary } \\
\text { substance P (SP) } \\
\text { levels. SP is known to } \\
\text { act as a } \\
\text { neurotransmitter in the } \\
\text { swallowing reflex. Low } \\
\text { levels of SP in saliva } \\
\text { attenuate the } \\
\text { swallowing reflex. SSF } \\
\text { data collected for 1-hr } \\
\text { per condition. }\end{array}$ & $\begin{array}{l}\text { Microphone } \\
\text { placed onto the } \\
\text { anterolateral side } \\
\text { of the neck. }\end{array}$ \\
\hline $\begin{array}{l}\text { Murray et } \\
\text { al. } 1996 \\
\text { [13] }\end{array}$ & $\begin{array}{l}\text { Elderly, } \\
\text { hospitalise } \\
\text { Elderly, normal } \\
\text { Young, normal }\end{array}$ & $\begin{array}{l}47 \\
29 \\
18\end{array}$ & $\begin{array}{l}\frac{0.89}{( \pm 0.85)} \\
\text { sw/min } \\
\text { Asp: } 0.72 \\
( \pm 0.78) \\
\text { sw/min } \\
\\
\text { No asp: } \\
1.16 \\
( \pm 0.91) \\
\text { sw/min } \\
\\
2.82 \\
( \pm 1.71) \\
\text { sw/min } \\
2.96 \\
( \pm 0.88) \\
\text { sw/min }\end{array}$ & 0.047 & $\begin{array}{l}\text { Investigation of SSF in } \\
\text { the predication of } \\
\text { aspiration of food and } \\
\text { liquid, following } \\
\text { dysphagia } \\
\text { categorisation using } \\
\text { fiberoptic endoscopic } \\
\text { evaluation of } \\
\text { swallowing (FEES). }\end{array}$ & $\begin{array}{l}\text { Fiberoptic } \\
\text { endoscopic } \\
\text { evaluation of } \\
\text { swallowing } \\
\text { (FEES). }\end{array}$ \\
\hline
\end{tabular}




\begin{tabular}{|c|c|c|c|c|c|c|}
\hline $\begin{array}{l}\text { Author, } \\
\text { year }\end{array}$ & Aetiology & $n$ & $\begin{array}{l}\text { Swallow } \\
\text { rate }\end{array}$ & $P$ & Additional details & $\begin{array}{l}\text { Swallow } \\
\text { identification } \\
\text { method }\end{array}$ \\
\hline \multirow{2}{*}{$\begin{array}{l}\text { Crary et al. } \\
2013 \\
{[14]}\end{array}$} & Elderly, normal & 29 & \multirow{2}{*}{$\begin{array}{l}0.47 \\
\mathrm{sw} / \mathrm{min} \\
1.02 \\
\mathrm{sw} / \mathrm{min}\end{array}$} & \multirow[t]{2}{*}{$<0.0001$} & \multirow[b]{2}{*}{$\begin{array}{l}\text { To evaluate an } \\
\text { acoustic recording } \\
\text { technique as a } \\
\text { measure to estimate } \\
\text { SSF. No significant } \\
\text { differences in } \\
\text { spontaneous swallow } \\
\text { frequency were } \\
\text { observed between the } \\
\text { multichannel } \\
\text { physiologic recordings } \\
\text { and the acoustic } \\
\text { recordings }(0.85 \text { vs. } \\
0.81 \mathrm{sw} / \mathrm{min}) \text {. }\end{array}$} & \multirow[b]{2}{*}{$\begin{array}{l}\text { Multichannel } \\
\text { recordings } \\
\text { including: surface } \\
\text { EMG, swallow } \\
\text { apnoea, and } \\
\text { cervical } \\
\text { auscultation. } \\
\text { Microphone for } \\
\text { acoustic } \\
\text { recordings } \\
\text { attached just } \\
\text { below the lateral } \\
\text { cricoid cartilage. }\end{array}$} \\
\hline & Young, normal & 18 & & & & \\
\hline \multirow{5}{*}{$\begin{array}{l}\text { Tanaka et } \\
\text { al. } 2013 \\
\text { [15] }\end{array}$} & Elderly, normal & $\underline{20}$ & $\frac{0.16}{(+0.08)}$ & \multirow{3}{*}{0.023} & \multirow{5}{*}{$\begin{array}{l}\text { SSF in elderly people } \\
\text { during daily life: a } \\
\text { comparison of (1) } \\
\text { elderly versus young, } \\
\text { and (2) elderly } \\
\text { bedridden versus } \\
\text { elderly semi-bedridden. } \\
\text { Recorded for 1-hr each } \\
\text { time. }\end{array}$} & \multirow{5}{*}{$\begin{array}{l}\text { Laryngeal } \\
\text { microphone and } \\
\text { digital voice } \\
\text { recorder. }\end{array}$} \\
\hline & Bedridden & 10 & $\mathrm{sw} / \mathrm{min}$ & & & \\
\hline & $\begin{array}{l}\text { Semi- } \\
\text { bedridden }\end{array}$ & 10 & $\begin{array}{l}0.11 \\
( \pm 0.06) \\
\mathrm{sw} / \mathrm{min}\end{array}$ & & & \\
\hline & \multirow[t]{2}{*}{ Young, normal } & 15 & $\begin{array}{l}0.2( \pm 0.09) \\
\mathrm{sw} / \mathrm{min}\end{array}$ & \multirow[t]{2}{*}{$<0.0001$} & & \\
\hline & & & $\begin{array}{l}0.68 \\
( \pm 0.33) \\
\text { sw/min }\end{array}$ & & & \\
\hline \multirow{2}{*}{$\begin{array}{l}\text { Trocello et } \\
\text { al. } 2015 \\
\text { [19] }\end{array}$} & \multirow[t]{2}{*}{$\begin{array}{l}\text { Wilson's } \\
\text { disease }\end{array}$} & \multirow{2}{*}{$\begin{array}{l}2 \\
6\end{array}$} & $\begin{array}{l}\text { Dys: } 0.97 \\
\text { sw/min }\end{array}$ & $>0.05$ & \multirow{2}{*}{$\begin{array}{l}\text { Examination of } \\
\text { hypersialorrhea in } \\
\text { Wilson's disease and } \\
\text { association with } \\
\text { dysphagia severity. } \\
\text { SSF recorded for } 10 \\
\text { mins. }\end{array}$} & \multirow{2}{*}{$\begin{array}{l}\text { Stethoscope } \\
\text { attached to the } \\
\text { neck and a } \\
\text { microphone } \\
\text { connected to a } \\
\text { rhinolaryngeal } \\
\text { stroboscope. }\end{array}$} \\
\hline & & & $\begin{array}{l}\text { Non-dys: } \\
1.35 \\
\text { sw/min }\end{array}$ & & & \\
\hline \multirow{7}{*}{$\begin{array}{l}\text { Kamarunas } \\
\text { et al. } 2018 \\
\text { [24] }\end{array}$} & $\begin{array}{l}\text { Oropharyngeal } \\
\text { dysphagia: }\end{array}$ & 13 & & & \multirow{7}{*}{$\begin{array}{l}\text { Group comparisons on } \\
\text { SSF without vibration } \\
\text { intervention. Data used } \\
\text { as baseline } \\
\text { information for study } \\
\text { aiming to evaluate } \\
\text { whether sensory } \\
\text { stimulation could } \\
\text { excite an impaired } \\
\text { swallowing system } \\
\text { (via use of SSF). }\end{array}$} & \multirow{3}{*}{$\begin{array}{l}\text { 1) Hyolaryngeal } \\
\text { elevation } \\
\text { (piezoelectric } \\
\text { accelerometer } \\
\text { peaks). }\end{array}$} \\
\hline & Post-CVA & \multirow{3}{*}{$\begin{array}{l}9 \\
4\end{array}$} & $\begin{array}{l}0.73( \pm 0.75) \\
\mathrm{sw} / \mathrm{min}\end{array}$ & & & \\
\hline & \multirow{3}{*}{$\begin{array}{l}\text { Post-radiation } \\
\text { (H/N cancer) }\end{array}$} & & $0.7( \pm 1.15)$ & & & \\
\hline & & & $\mathrm{sw} / \mathrm{min}$ & & & \multirow{4}{*}{$\begin{array}{l}\text { 2) Respiratory } \\
\text { apnoea (inductive } \\
\text { plethysmography } \\
\text { - absence of } \\
\text { ribcage/abdomen } \\
\text { movement). } \\
\text { 3) note from } \\
\text { trained observer. }\end{array}$} \\
\hline & & 10 & & 0.48 & & \\
\hline & \multirow{2}{*}{$\begin{array}{l}\text { Healthy } \\
\text { control (taken } \\
\text { from Mulheren } \\
\text { et al. } 2017\end{array}$} & & $\begin{array}{l}1.27 \\
( \pm 1.22)\end{array}$ & & & \\
\hline & & & & & & \\
\hline
\end{tabular}




\begin{tabular}{|c|c|c|c|c|c|c|}
\hline $\begin{array}{l}\text { Author, } \\
\text { year }\end{array}$ & Aetiology & $n$ & $\begin{array}{l}\text { Swallow } \\
\text { rate }\end{array}$ & $P$ & Additional details & $\begin{array}{l}\text { Swallow } \\
\text { identification } \\
\text { method }\end{array}$ \\
\hline \multirow{2}{*}{$\begin{array}{l}\text { Pehlivan et } \\
\text { al. } 1996 \\
\text { [26] }\end{array}$} & Parkinsons & 21 & $0.8 \mathrm{sw} / \mathrm{min}$ & \multirow[t]{2}{*}{$<0.05$} & \multirow{2}{*}{$\begin{array}{l}\text { Use of "Digital } \\
\text { Phagometer" } \\
\text { (piezoelectric sensor } \\
\text { and digital event } \\
\text { counter) to measure } \\
\text { SSF in patients with } \\
\text { Parkinsons. }\end{array}$} & \multirow{2}{*}{$\begin{array}{l}\text { Piezoelectric } \\
\text { sensor placed at } \\
\text { the coniotomy } \\
\text { region between } \\
\text { the thyroid and } \\
\text { cricoid cartilages. }\end{array}$} \\
\hline & $\begin{array}{l}\text { Healthy } \\
\text { control }\end{array}$ & 21 & $\begin{array}{l}1.18 \\
\mathrm{sw} / \mathrm{min}\end{array}$ & & & \\
\hline \multirow{3}{*}{$\begin{array}{l}\text { Marks \& } \\
\text { Weinreich. } \\
2001 \\
{[12]}\end{array}$} & Parkinsons & 28 & 0.55 & \multirow{3}{*}{$\begin{array}{l}\text { Not } \\
\text { reported }\end{array}$} & \multirow{3}{*}{$\begin{array}{l}\text { Use of an electret } \\
\text { microphone to } \\
\text { measure SSF to give } \\
\text { an indication of } \\
\text { drooling in patients } \\
\text { with Parkinsons. }\end{array}$} & \multirow{3}{*}{$\begin{array}{l}\text { Microphone } \\
\text { positioned over } \\
\text { the centre of the } \\
\text { cricoid cartilage. }\end{array}$} \\
\hline & \multirow{2}{*}{$\begin{array}{l}\text { Healthy, age- } \\
\text { matched } \\
\text { control }\end{array}$} & \multirow[t]{2}{*}{8} & $\mathrm{sw} / \mathrm{min}$ & & & \\
\hline & & & $\begin{array}{l}0.13 \\
( \pm 0.03) \\
\mathrm{sw} / \mathrm{min}\end{array}$ & & & \\
\hline \multirow{3}{*}{$\begin{array}{l}\text { Kalf et al. } \\
2011 \\
{[17]}\end{array}$} & $\begin{array}{l}\text { Parkinsons, } \\
\text { droolers }\end{array}$ & 15 & $\begin{array}{l}0.51 \\
( \pm 0.39)\end{array}$ & \multirow[t]{3}{*}{0.346} & \multirow{3}{*}{$\begin{array}{l}\text { Factors potentially } \\
\text { contributing to } \\
\text { drooling, including SSF, } \\
\text { examined in } \\
\text { Parkinsons patients } \\
\text { with and without } \\
\text { diurnal saliva loss. }\end{array}$} & \multirow{3}{*}{$\begin{array}{l}\text { EMG, motion } \\
\text { sensor (at larynx), } \\
\text { and video. }\end{array}$} \\
\hline & \multirow{2}{*}{$\begin{array}{l}\text { Parkinsons, } \\
\text { nondroolers }\end{array}$} & 15 & $\mathrm{sW} / \mathrm{mIn}$ & & & \\
\hline & & & $\begin{array}{l}0.4( \pm 0.26) \\
\mathrm{sw} / \mathrm{min}\end{array}$ & & & \\
\hline
\end{tabular}

Table 2. Observational studies measuring spontaneous swallow frequency in patients with, or at risk of developing, dysphagia 


\begin{tabular}{|c|c|c|c|c|c|c|}
\hline $\begin{array}{l}\text { Author, } \\
\text { year }\end{array}$ & Aetiology & $n$ & Swallow rate & $P$ & $\begin{array}{l}\text { Additional } \\
\text { details }\end{array}$ & $\begin{array}{l}\text { Swallow } \\
\text { identification } \\
\text { method }\end{array}$ \\
\hline $\begin{array}{l}\text { Brady et al. } \\
2016 \\
{[23]}\end{array}$ & $\begin{array}{l}\text { Known or } \\
\text { suspected } \\
\text { dysphagia }\end{array}$ & 27 & $\begin{array}{l}\text { OSL: } \\
\text { - Normal/mild: } \\
\text { Pre-GS: } 0.53 \\
\text { Post-GS: } 2.63 \\
\text { sw/min } \\
\text { - } \\
\text { Severe/profound: } \\
\text { Pre-GS: } 0.06 \\
\text { Post-GS: } 1.75 \\
\text { sw/min }\end{array}$ & & $\begin{array}{l}\text { Investigation of } \\
\text { the relationship } \\
\text { between SSF, } \\
\text { accumulated } \\
\text { oropharyngeal } \\
\text { secretion levels } \\
\text { (OSL), and } \\
\text { gustatory } \\
\text { stimulation } \\
\text { (GS). Moderate } \\
\text { relationship } \\
\text { between SSF at } \\
\text { rest and OSL } \\
\text { (Pearson } \\
\text { correlation } \\
0.47 ; p=0.01) .\end{array}$ & $\begin{array}{l}\text { Visually by two } \\
\text { raters (inspection } \\
\text { of larynx) with } \\
\text { assistance from } \\
\text { surface EMG. }\end{array}$ \\
\hline $\begin{array}{l}\text { Konradi et } \\
\text { al. } 2015 \\
{[21]}\end{array}$ & $\begin{array}{l}\text { Acute } \\
\text { neurogenic } \\
\text { dysphagia }\end{array}$ & 19 & $\begin{array}{l}\text { Pre: } 0.28( \pm 0.26) \\
\text { sw/min } \\
\text { During } \\
\text { intervention: } 0.47 \\
( \pm 0.32) \mathrm{sw} / \mathrm{min} \\
\text { Follow- up }(5 \\
\text { mins later }): 0.33 \\
( \pm 0.28) \mathrm{sw} / \mathrm{min}\end{array}$ & $\begin{array}{l}0.037 \\
0.44\end{array}$ & $\begin{array}{l}\text { To investigate } \\
\text { the direct effect } \\
\text { of facio-oral } \\
\text { tract therapy } \\
\text { on SSF of non- } \\
\text { tracheotomised } \\
\text { patients with } \\
\text { acute } \\
\text { neurogenic } \\
\text { dysphagia. }\end{array}$ & $\begin{array}{l}\text { Visually and by } \\
\text { palpation during } \\
\text { laryngeal elevation. }\end{array}$ \\
\hline $\begin{array}{l}\text { Kothari et } \\
\text { al. } 2017 \\
\text { [27] }\end{array}$ & $\begin{array}{l}\text { Brain injury, } \\
\text { with } \\
\text { dysphagia }\end{array}$ & 10 & $\begin{array}{l}\text { Baseline: } 0.12 \\
( \pm 0.05) \mathrm{sw} / \mathrm{min} \\
\text { During-ESAF: } \\
0.42( \pm 0.14) \\
\mathrm{sw} / \mathrm{min}\end{array}$ & $<0.001$ & $\begin{array}{l}\text { To determine if } \\
\text { external } \\
\text { subglottic air } \\
\text { flow (ESAF) } \\
\text { influences } \\
\text { swallowing } \\
\text { frequency in } \\
\text { severely } \\
\text { dysphagic } \\
\text { tracheotomised } \\
\text { patients with } \\
\text { brain injury. }\end{array}$ & $\begin{array}{l}\text { Visually by } \\
\text { occupational } \\
\text { therapists. }\end{array}$ \\
\hline
\end{tabular}




\begin{tabular}{|c|c|c|c|c|c|c|}
\hline $\begin{array}{l}\text { Author, } \\
\text { year }\end{array}$ & Aetiology & $n$ & Swallow rate & $P$ & $\begin{array}{l}\text { Additional } \\
\text { details }\end{array}$ & $\begin{array}{l}\text { Swallow } \\
\text { identification } \\
\text { method }\end{array}$ \\
\hline $\begin{array}{l}\text { Seidl et al. } \\
2005 \\
{[28]}\end{array}$ & $\begin{array}{l}\text { Neurogenic } \\
\text { dysphagia }\end{array}$ & 10 & $\begin{array}{l}\text { With TT: } 0.08 \\
( \pm 0.16) \mathrm{sw} / \mathrm{min} \\
\text { Without TT: } 0.33 \\
( \pm 0.3) \mathrm{sw} / \mathrm{min}\end{array}$ & 0.001 & $\begin{array}{l}\text { Comparing } \\
\text { SSF in patients } \\
\text { with } \\
\text { neurogenic } \\
\text { dysphagia with } \\
\text { or without } \\
\text { tracheotomy } \\
\text { tubes (TT) to } \\
\text { assess the } \\
\text { underlying } \\
\text { mechanisms of } \\
\text { dysphagia to } \\
\text { improve } \\
\text { rehabilitation } \\
\text { strategies. SSF } \\
\text { was assessed } \\
\text { by counting } \\
\text { elevation of the } \\
\text { larynx over 5- } \\
\text { min. }\end{array}$ & $\begin{array}{l}\text { Observations were } \\
\text { videotaped and } \\
\text { evaluated by } 2 \\
\text { independent } \\
\text { investigators. }\end{array}$ \\
\hline $\begin{array}{l}\text { Seidl et al. } \\
2007 \\
{[22]}\end{array}$ & $\begin{array}{l}\text { Neurological } \\
\text { disorders }\end{array}$ & 10 & $\begin{array}{l}\text { Baseline (day } 1) \text { : } \\
0.09( \pm 0.2) \\
\text { sw/min } \\
\text { Final day (day } \\
15): 0.47( \pm 0.35) \\
\text { sw/min }\end{array}$ & 0.043 & $\begin{array}{l}\text { To investigate } \\
\text { the success of } \\
\text { facio-oral } \\
\text { therapy in } \\
\text { patients with } \\
\text { neurological } \\
\text { disorders. SSF } \\
\text { was assessed } \\
\text { by counting } \\
\text { elevation of the } \\
\text { larynx. }\end{array}$ & $\begin{array}{l}\text { Observations were } \\
\text { videotaped and } \\
\text { evaluated by } 2 \\
\text { independent } \\
\text { investigators. }\end{array}$ \\
\hline $\begin{array}{l}\text { Kamarunas } \\
\text { et al. 2018* } \\
\text { [24] }\end{array}$ & $\begin{array}{l}\text { Oropharyngeal } \\
\text { dysphagia }\end{array}$ & 13 & $\begin{array}{l}\text { Sw/min raw data } \\
\text { not reported } \\
\text { (only shown in } \\
\text { table) } \\
\text { Sham vs.: } \\
30 \mathrm{~Hz} \\
70 \mathrm{~Hz} \\
110 \mathrm{~Hz} \\
70+100 \mathrm{~Hz} \\
150 \mathrm{~Hz}\end{array}$ & $\begin{array}{l}<0.001 \\
>0.025 \\
<0.001 \\
>0.025 \\
>0.025\end{array}$ & $\begin{array}{l}\text { To examine if } \\
\text { laryngeal } \\
\text { stimulation } \\
\text { increases SSF, } \\
\text { and if so, } \\
\text { discover the } \\
\text { optimal rate of } \\
\text { vibration using } \\
\text { a device. The } \\
\text { device showed } \\
\text { the potential to } \\
\text { increase SSF at } \\
70 \mathrm{~Hz} \text { and } \\
110 \mathrm{~Hz} \\
\text { frequencies. }\end{array}$ & $\begin{array}{l}\text { 1) Hyolaryngeal } \\
\text { elevation } \\
\text { (piezoelectric } \\
\text { accelerometer } \\
\text { peaks). } \\
\text { 2) Respiratory } \\
\text { apnoea (inductive } \\
\text { plethysmography - } \\
\text { absence of } \\
\text { ribcage/abdomen } \\
\text { movement). } \\
\text { 3) Note from } \\
\text { trained observer. }\end{array}$ \\
\hline
\end{tabular}




\begin{tabular}{|c|c|c|c|c|c|c|}
\hline $\begin{array}{l}\text { Author, } \\
\text { year }\end{array}$ & Aetiology & $n$ & Swallow rate & $P$ & $\begin{array}{l}\text { Additional } \\
\text { details }\end{array}$ & $\begin{array}{l}\text { Swallow } \\
\text { identification } \\
\text { method }\end{array}$ \\
\hline $\begin{array}{l}\text { Mulheren } \\
\text { et al. } 2017 \\
\text { [29] }\end{array}$ & Healthy adults & 10 & $\begin{array}{l}\text { Sw/min raw data } \\
\text { not reported - } \\
\text { instead SSF } \\
\text { reported as } Z \\
\text { scores } \\
\text { Sham vs.: } \\
30 \mathrm{~Hz} \\
70 \mathrm{~Hz} \\
110 \mathrm{~Hz} \\
70+100 \mathrm{~Hz} \\
150 \mathrm{~Hz}\end{array}$ & $\begin{array}{l}>0.05 \\
0.05 \\
>0.05 \\
>0.05 \\
0.043\end{array}$ & $\begin{array}{l}\text { To determine if } \\
\text { vibration in } \\
\text { comparison to } \\
\text { a sham device } \\
\text { increases SSF } \\
\text { and enhances } \\
\text { cortical } \\
\text { hemodynamic } \\
\text { responses to } \\
\text { swallows. SSF } \\
\text { analysed over } \\
\text { 20-min periods } \\
\text { in each } \\
\text { condition. }\end{array}$ & $\begin{array}{l}\text { 1) Hyolaryngeal } \\
\text { elevation (rapid } \\
\text { accelerometer } \\
\text { change). } \\
\text { 2) Respiratory } \\
\text { apnoea (inductive } \\
\text { plethysmography - } \\
\text { absence of } \\
\text { ribcage/abdomen } \\
\text { movement). } \\
\text { 3) Note from } \\
\text { trained observer. }\end{array}$ \\
\hline $\begin{array}{l}\text { Theurer et } \\
\text { al. } 2005 \\
\text { [30] }\end{array}$ & Healthy adults & 4 & $\begin{array}{l}\text { Baseline: } 0.86 \\
( \pm 0.18) \mathrm{sw} / \mathrm{min} \\
\text { Unilateral (R): } \\
1.95( \pm 0.89) \\
\text { sw/min } \\
\text { Unilateral (L): } \\
\text { Not reported raw } \\
(\sim 1.6 \pm 0.18) \\
\text { sw/min } \\
\text { Bilateral: } 2.35 \\
( \pm \mathrm{R} 1.32) \mathrm{sw} / \mathrm{min} \\
\text { Hand: } 0.55( \pm \\
0.38) \mathrm{sw} / \mathrm{min}\end{array}$ & & $\begin{array}{l}\text { To determine } \\
\text { whether air- } \\
\text { pulse trains } \\
\text { delivered to the } \\
\text { peritonsillar } \\
\text { area facilitate } \\
\text { swallowing in } \\
\text { adults - } \\
\text { measured as } \\
\text { SSF over a 5- } \\
\text { min period. }\end{array}$ & $\begin{array}{l}\text { Laryngeal and } \\
\text { respiratory } \\
\text { movements using } \\
\text { pressure } \\
\text { transducers from } \\
\text { expanding bellows. }\end{array}$ \\
\hline $\begin{array}{l}\text { South et al. } \\
2010 \\
{[18]}\end{array}$ & Parkinsons & 23 & $\begin{array}{l}\text { Baseline: } 0.62 \\
( \pm 0.57) \text { sw/min } \\
\text { GC: } 2.99( \pm 0.6) \\
\text { sw/min } \\
\text { Post-GC: } 1.4 \\
( \pm 0.51) \mathrm{sw} / \mathrm{min}\end{array}$ & $\begin{array}{l}<0.0001 \\
<0.0011\end{array}$ & $\begin{array}{l}\text { Assessing the } \\
\text { swallow } \\
\text { frequency of } \\
\text { patients with } \\
\text { Parkinsons at: } \\
\text { baseline, } \\
\text { during gum } \\
\text { chewing (GC), } \\
\text { and post-CG (5- } \\
\text { min each). }\end{array}$ & $\begin{array}{l}\text { Laryngeal bellows } \\
\text { and respiratory } \\
\text { bellows. }\end{array}$ \\
\hline
\end{tabular}




\begin{tabular}{|c|c|c|c|c|c|c|}
\hline $\begin{array}{l}\text { Author, } \\
\text { year }\end{array}$ & Aetiology & $n$ & Swallow rate & $P$ & $\begin{array}{l}\text { Additional } \\
\text { details }\end{array}$ & $\begin{array}{l}\text { Swallow } \\
\text { identification } \\
\text { method }\end{array}$ \\
\hline $\begin{array}{l}\text { D'Angelo et } \\
\text { al. } 2014 \\
\text { [31] }\end{array}$ & $\begin{array}{l}\text { Healthy adults } \\
\text { (vs. nocturnal) }\end{array}$ & 11 & $\begin{array}{l}\text { Under } \\
\text { anaesthesia: } \\
0.03( \pm 0.06) \\
\text { vs. Awake: } 0.47 \\
( \pm 0.37) \text { sw/min } \\
\text { Under } \\
\text { anaesthesia: } \\
0.02( \pm 0.09) \\
\text { vs. } \\
+ \text { Hypercapnia: } \\
0.9( \pm 0.28) \\
\text { sw/min } \\
\text { Awake: } 0.5( \pm 0.4) \\
\text { vs. } \\
+ \text { Hypercapnia: } \\
0.37( \pm 0.4) \\
\text { sw/min }\end{array}$ & $\begin{array}{l}<0.001 \\
0.006 \\
>0.05\end{array}$ & $\begin{array}{l}\text { Examining the } \\
\text { effects of } \\
\text { anaesthesia } \\
\text { and } \\
\text { hypercapnia on } \\
\text { swallowing- } \\
\text { breathing } \\
\text { coordination. }\end{array}$ & $\begin{array}{l}\text { 1) Pressure } \\
\text { catheter (inserted } \\
\text { nasally into the } \\
\text { hypopharyngeal } \\
\text { area). } \\
\text { 2) EMG (using wire } \\
\text { electrodes inserted } \\
\text { into the } \\
\text { genioglossus } \\
\text { muscle). } \\
\text { 3) } \\
\text { Pneumotachograph } \\
\text { (to measure } \\
\text { respiratory flow). }\end{array}$ \\
\hline
\end{tabular}

*Repeated in both tables as paper included data from both observational and interventional sub-studies.

Table 3. Interventional studies measuring spontaneous swallow frequency in patients with, or at risk of developing, dysphagia

\section{Study characteristics}

\section{Study design}

The 19 papers reported a mixture of observational ( $n=9$ case-control or cross-sectional) and interventional $(n=$ 10, non-randomised pre-post intervention) studies. As such, five were rated of good quality, six as fair/good quality, seven as fair quality, with a single paper of poor quality (Appendix A). The raters agreed that the poor quality paper [12] failed in a number of key areas, including: an inadequate definition of the study population; no sample size justification; high risk of researcher bias due to lack of blinding or confounding variables; and insufficient detail with regard to the study controls and inclusion/exclusion criteria.

Small groups of highly disparate aetiologies were reported, often $n=10$ or fewer with the largest single group being $\mathrm{n}=47$. This increases the likelihood that significant comorbidities be present in some groups and not in others. For example, hypersialorrhea is prevalent in patients with Parkinson's disease (PD) and Wilson's disease, which may increase swallowing frequency even in those with swallowing difficulties.

\section{Swallow assessment methods}


Tables 2 and 3 present detail of the different swallow assessments, and ultimately show that there is no standardised way in which to measure spontaneous swallowing. There was little consistency in identification of persons with dysphagia, timing of observations, or methods for detecting swallows. There was a single report (Murray et al. [13]) of Fibre-endoscopic Evaluation of Swallow (FEES). We excluded Murray's data from metaanalysis because the FEES investigation is invasive; as we explain later, this likely cannot be considered comparable with the other non-invasive methods.

\section{Meta-analysis}

For 30 groups in 19 papers there was an estimate of spontaneous swallow frequency in either a healthy or clinically relevant population (Tables 2 and 3). The meta-analysis is presented as a Forest plot in Figure 2 . The outcome measure MLN is the log transformed (geometric) mean of the groups from the relevant studies.

\section{Heterogeneity}

The estimates of SSF are visibly variable between and within all groups (Figure 2). Therefore we report high heterogeneity overall $\left(\mathrm{I}^{2}=98 \%, \mathrm{t}^{2}=0.56\right)$ and also within each sub-group (dysphagic, $\mathrm{I}^{2}=86 \%$; Parkinson's, $\mathrm{I}^{2}=$ $92 \%$; higher risk, $\mathrm{I}^{2}=95 \%$; healthy older, $\mathrm{I}^{2}=97 \%$; healthy younger, $\mathrm{I}^{2}=89 \%$ ). This implies that the large differences in SSF between studies cannot be explained by random variability alone.

\section{Estimate of spontaneous swallow frequency}

The best estimate of overall SSF is $0.41[0.31 ; 0.54] / \mathrm{min}$. The high heterogeneity will be explained at least in part by the anticipated differences between the sub-groups. To consider the sub-groups, the range of SSF in healthy younger was $0.47-1.7$ / min, healthy older 0.13-0.47, Parkinson's 0.4-0.92, higher risk (i.e. post-stroke, bedridden, neurogenic swallow disorders) $0.09-0.55$ and Dysphagic 0.06-0.73. Notably Murray et al. [13] with his instrumental FEES assessment reported a dramatically higher swallowing rate in both healthy young (SSF: 2.82 [ \pm 1.71$])$ and healthy older (SSF: $2.96[ \pm 0.88])$ volunteers than any other group; see the discussion.

Turning to the meta-analysis, SSF was similar in dysphagic $(0.27[0.17 ; 0.43])$, higher risk $(0.26[0.14 ; 0.85])$, and healthy older (0.21 [0.12;0.37]) groups. Whereas, Parkinson's groups had an SSF of $0.59[0.41 ; 0.85]$ and healthy younger groups average SSF was higher still at $0.98[0.78 ; 1.23]$.

\section{Discussion}

There is insufficient evidence to describe normative values for SSF in either healthy populations or clinically relevant groups. It is also not possible to determine whether SSF is a clinically useful screening tool for dysphagia at present. It was clear from the analysis that data on swallow frequency from different centres, using different technologies or different methodologies, cannot reliably be compared. However, from the intervention studies in table 3, it appears that differences in a swallow index measured in the same centre using the same study design are sensitive to clinical condition or to intervention. This seems reasonable since in this situation the major methodological differences are controlled. Nevertheless the relation of any measure of SSF with a physiologically meaningful reference standard cannot be assumed.

Page $14 / 22$ 


\section{Effect of age and gender on spontaneous swallow frequency}

Despite high heterogeneity, there is a negative correlation of SSF with age. Healthy young ( $<60$ years) have a mean SSF of 0.98 while healthy old have a mean SSF of 0.21 . Simply, older people swallow less. This is in agreement with the works of Crary et al. [14] and Tanaka et al. [15], both of whom report significantly higher SSF in young normal (healthy) subjects when compared to elderly normal (healthy) subjects using the same swallow detection technology (see Table 2). However, note that mean SSFs across even the healthy young group are highly variable and do not give a reliable indication of what a true 'normal' SSF is.

As with all correlation studies, cause and effect is difficult to assess. The reduced SSF with advanced age may be directly implicated in dysphagia. If so, it is surprising that SSF is so similar across the elderly population, irrespective of dysphagia risk. If some other age-related process causes dysphagia then SSF is merely a convoluted method of estimating age.

Finally, one conference abstract reported a higher rate of spontaneous swallow in women [16]. This is an important area to investigate, but no full papers have yet been published assessing the effect of gender on SSF.

\section{The role of spontaneous swallow frequency in clinical practice}

Accurate measurement of SSF may be the most appropriate reference for basic research in swallow physiology. If instead we consider the diagnosis of dysphagia as our criterion, then a validated clinical assessment by SALT may be a more appropriate reference standard. Crary et al. [7] compared SSF to a clinical assessment, reporting that a threshold of 0.4 swallows/min showed high sensitivity (0.96) but low specificity (0.68) for dysphagia. The workers suggest SSF may be a useful screening tool but lacks the specificity for a definitive diagnosis. Our data would agree; those at heightened risk of dysphagia have a much lower SSF than younger healthy participants. With the exception of PD, the mean SSF was in every other case lower than Crary's threshold. Note however that this is the mean SSF; sensitivity and specificity are defined on a patient-by-patient level.

\section{Secretion management and interventions to increase SSF}

Interventions to increase swallowing frequency have been used to aid in secretion management and have increased understanding in conditions where hypersalivation and resultant drooling is common, namely, Parkinson's $[17,18]$ and Wilson's Disease [19]. PD is also associated with increased risk of dysphagia. More than $80 \%$ of patients are predicted to develop dysphagia following disease onset [20]. Figure 3 suggests that PD patients have a lower SSF than healthy younger subjects but they appear to swallow more regularly than other atrisk groups. This may be explained by known and unknown cases of hypersalivation in this patient population [17]. Moreover, SSF reported across studies involving PD patients is highly variable, potentially due, in part, to the risk of associated conditions such as dysphagia that have not been diagnosed. Such conflicting variables make it difficult to determine the true value of using SSF in this population.

Interventions to stimulate swallows appeared to be successful though these studies were often not blinded or randomised so were open to bias, and it is difficult to know to what degree any improvements could be considered clinically significant. Four studies were effective at increasing SSF in groups with dysphagia; two found facio-oral tract therapy to significantly increase swallow rate [21, 22] one used gustatory stimulation [23], and one 
successfully applied vibrations to stimulation the larynx in a dysphagic group [24]. Studies reported that these interventions have the potential to improve swallow function in those with dysphagia via the measurement of SSF, but no studies report long term outcomes.

It remains unclear what a normative swallow rate is and what a clinically significant change in rate may be. Nevertheless he pre- and post- intervention data reported maybe give useful insight for researchers and clinicians to consider in the future when treating these patient groups.

\section{SSF measurement methodologies}

Heterogeneity between studies was high, particularly with regard to methods used to assess SSF. A variety of techniques were used to measure spontaneous swallows, most frequently with the single or combined use of: electromyography (EMG), acoustic/sound recording, respiratory bellows/transducers (around the ribcage), bellows/transducers located around the larynx, or scoring by a trained observer. Other techniques included auscultation, fiberoptic endoscopic evaluation (FEES), and magnetic resonance imaging (MRI).

A clinically useful measure of SSF should have good validity, i.e. should give comparable results to a reference standard. A reference for the measurement of SSF (i.e. the detection of swallows over prolonged periods of time) does not currently exist. Nevertheless any clinical physiological measurement - sphygmomanometry for example - is predicated on the idea that the measurement indicates the underlying blood pressure irrespective of the specific technology. There is general consensus as to what constitutes a swallow, and so there is objectively a 'correct' SSF, at least in principle. If measurements disagree to the extent reported here, we cannot avoid the conclusion that some (or possibly all) technologies are generating incorrect data.

To resolve these issues, comparative studies between several measures of SSF are needed. Only one group compared two systems for measuring SSF [14], the first an acoustic recording technique, the comparator a comprehensive multichannel physiologic recording with surface EMG, swallow apnoea, and cervical auscultation. There was no significant difference in SSF between the two methods $(0.85 / \mathrm{min} v \mathrm{vs}$. $0.81 / \mathrm{min})$. Superficially this suggests that a microphone alone might be used to record swallows. Perhaps more importantly, the consistency between two different technologies might suggest that the technology works well and that other methodological factors (for example: the use of long-term ambulatory use versus lab-based investigations with prescribed activities) is responsible for the poor agreement between centres.

FEES is a well-used and reliable method for assessing a single swallow and may be an appropriate reference standard for an event-by-event comparison, a form of criterion validity. Large scale validation studies of SSF instrumentation are required before systematic trials in clinical populations, with data involving known swallows and potential artefacts. In order to ensure high specificity with regards to swallow detection, designs must rule out noise artefacts in addition to reliably identifying all swallows.

\section{Conclusion}

SSF is a novel, non-invasive clinical variable which warrants further explorations as to its potential to identify persons at risk of dysphagia. Larger, well-conducted studies are needed to develop objective, standardised methods for detecting SSF, and develop normative values in healthy populations in order to answer this question. 


\section{Abbreviations}

$\mathrm{Cl}$ - Confidence interval

EMG - Electromyography

FEES - Fiberoptic endoscopic evaluation of swallowing

HCAI - Healthcare-associated infection

HES - Health Episode Statistics

MLN - Log transformed means

MRI - Magnetic resonance imaging

PD - Parkinson's disease

SALT - Speech and Language Therapy

SSF - Spontaneous Swallow frequency

\section{Declarations}

\section{Ethics approval and consent to participate}

Not applicable.

\section{Consent for publication}

Not applicable.

\section{Availability of data and materials}

The data that support the findings of this study are available on the public domain. See 'Search strategy and selection criteria' within the Method section on this paper for more details, including the literature search domains/databases used.

\section{Competing interests}

The authors declare that they have no competing interests.

\section{Funding}

Not applicable. 


\section{Authors' contributions}

VE and JB conceived the research question and developed the search strategy. JB conducted the search, and extracted and collated the metadata. MD and JB conducted the statistical analysis on the metadata. CE and JB conducted the quality assessment of the selected studies. JB, VE and MD reviewed and finalised the manuscript.

\section{Acknowledgements}

Not applicable.

\section{References}

1. Shaker, R. (1995). Airway protective mechanisms: current concepts. Dysphagia, 10(4), 216-227.

2. Vergis, E.N., C. Brennen, M. Wagener, and R.R. Muder, Pneumonia in long-term care: a prospective case-control study of risk factors and impact on survival. Archives of Internal Medicine, 2001. 161(19): p. 2378-81.

3. Harkness, G.A., D.W. Bentley, and K.J. Roghmann, Risk factors for nosocomial pneumonia in the elderly. American Journal of Medicine, 1990. 89(4): p. 457-63.

4. European Centre for Disease Prevention and Control, Point prevalence survey of healthcare-associated infections and antimicrobial use in European acute care hospitals 2011-2012

5. Etges, C. L., Scheeren, B., Gomes, E., \& Barbosa, L. D. R. (2014, October). Screening tools for dysphagia: a systematic review. In Codas (Vol. 26, No. 5, pp. 343-349). Sociedade Brasileira de Fonoaudiologia.

6. Park, Y. H., Bang, H. L., Han, H. R., \& Chang, H. K. (2015). Dysphagia screening measures for use in nursing homes: a systematic review. Journal of Korean Academy of Nursing, 45(1), 1-13.Pehlivan, M., Yüceyar, N., Ertekin, C., Çelebi, G., Ertaş, M., Kalayci, T., \& Aydoğdu, I. (1996). An electronic device measuring the frequency of spontaneous swallowing: digital phagometer. Dysphagia, 11(4), 259-264.

7. Crary, M. A., Carnaby, G. D., \& Sia, I. (2014). Spontaneous swallow frequency compared with clinical screening in the identification of dysphagia in acute stroke. Journal of Stroke and Cerebrovascular Diseases, 23(8), 2047-2053.

8. Lear, C. S., Flanagan Jr, J. B., \& Moorrees, C. F. A. (1965). The frequency of deglutition in man. Archives of oral biology, 10(1), 83-IN15.

9. Rudney, J. D., Ji, Z., \& Larson, C. J. (1995). The prediction of saliva swallowing frequency in humans from estimates of salivary flow rate and the volume of saliva swallowed. Archives of oral biology, 40(6), 507-512.

10. National Institutes of Health. (2014). Study Quality Assessment Tools| National Heart, Lung, and Blood Institute (NHLBI). National Institutes of Health.

11. R Core Team. R: A Language and Environment for Statistical Computing. Vienna, Austria: R Foundation for Statistical Computing. 2017. https://www.R-project.org/.

12. Marks, L., \& Weinreich, J. (2001). Drooling in Parkinson's disease: a novel tool for assessment of swallow frequency. International journal of language \& communication disorders, 36(sup1), 288-291.

13. Murray, J., Langmore, S. E., Ginsberg, S., \& Dostie, A. (1996). The significance of accumulated oropharyngeal secretions and swallowing frequency in predicting aspiration. Dysphagia, 11(2), 99-103. 
14. Crary, M. A., Sura, L., \& Carnaby, G. (2013). Validation and demonstration of an isolated acoustic recording technique to estimate spontaneous swallow frequency. Dysphagia, 28(1), 86-94.

15. Tanaka, N., Nohara, K., Kotani, Y., Matsumura, M., \& Sakai, T. (2013). Swallowing frequency in elderly people during daily life. Journal of oral rehabilitation, 40(10), 744-750.

16. Bradley, J. A., Paulson, E. S., Kharofa, J., Li, X., Schultz, C., \& Wang, D. (2012). Patient and Tumor Characteristics and Pretreatment Swallowing Frequency and Duration in Head-and-Neck Cancer Patients. International Journal of Radiation Oncology• Biology• Physics, 84(3), S512.

17. Kalf, J. G., Munneke, M., van den Engel-Hoek, L., de Swart, B. J., Borm, G. F., Bloem, B. R., \& Zwarts, M. J. (2011). Pathophysiology of diurnal drooling in Parkinson's disease. Movement disorders, 26(9), 1670-1676.

18. South, A. R., Somers, S. M., \& Jog, M. S. (2010). Gum chewing improves swallow frequency and latency in Parkinson patients: a preliminary study. Neurology, 74(15), 1198-1202.

19. Trocello, J. M., Osmani, K., Pernon, M., Chevaillier, G., de Brugiere, C., Remy, P., ... \& Woimant, F. (2015). Hypersialorrhea in Wilson's disease. Dysphagia, 30(5), 489-495.

20. Suttrup, I., \& Warnecke, T. (2016). Dysphagia in Parkinson's disease. Dysphagia, 31(1), 24-32.

21. Konradi, J., Lerch, A., Cataldo, M., \& Kerz, T. (2015). Direct effects of Facio-Oral Tract Therapy® on swallowing frequency of non-tracheotomised patients with acute neurogenic dysphagia. SAGE open medicine, 3 , 2050312115578958.

22. Seidl, R. O., Nusser-Müller-Busch, R., Hollweg, W., Westhofen, M., \& Ernst, A. (2007). Pilot study of a neurophysiological dysphagia therapy for neurological patients. Clinical rehabilitation, 21(8), 686-697.

23. Brady, S. L., Wesling, M. W., Donzelli, J. J., \& Kaszuba, S. (2016). Swallowing frequency: impact of accumulated oropharyngeal secretion levels and gustatory stimulation. Ear, Nose \& Throat Journal, 95(2), E7E9.

24. Kamarunas, E., Wong, S. M., \& Ludlow, C. L. (2018). Laryngeal Vibration Increases Spontaneous Swallowing Rates in Chronic Oropharyngeal Dysphagia: A Proof-of-Principle Pilot Study. Dysphagia, 1-14.

25. Niimi, M., Hashimoto, G., Hara, T., Yamada, N., Abo, M., Fujigasaki, H., \& Ide, T. (2018). Relationship Between Frequency of Spontaneous Swallowing and Salivary Substance P Level in Patients with Acute Stroke. Dysphagia, 33(4), 414-418.

26. Pehlivan, M., Yüceyar, N., Ertekin, C., Çelebi, G., Ertaş, M., Kalayci, T., \& Aydoğdu, I. (1996). An electronic device measuring the frequency of spontaneous swallowing: digital phagometer. Dysphagia, 11(4), 259-264.

27. Kothari, M., Bjerrum, K., Nielsen, L. H., Jensen, J., \& Nielsen, J. F. (2017). Influence of External Subglottic Air Flow on Dysphagic Tracheotomized Patients With Severe Brain Injury: Preliminary Findings. Annals of Otology, Rhinology \& Laryngology, 126(3), 199-204.

28. Seidl, R. O., Nusser-Müller-busch, R., \& Ernst, A. (2005). The influence of tracheotomy tubes on the swallowing frequency in neurogenic dysphagia. Otolaryngology-Head and Neck Surgery, 132(3), 484-486.

29. Mulheren, R. W., \& Ludlow, C. L. (2017). Vibration over the larynx increases swallowing and cortical activation for swallowing. Journal of neurophysiology, 118(3), 1698-1708.

30. Theurer, J. A., Bihari, F., Barr, A. M., \& Martin, R. E. (2005). Oropharyngeal stimulation with air-pulse trains increases swallowing frequency in healthy adults. Dysphagia, 20(4), 254-260.

31. D’Angelo, O. M., Diaz-Gil, D., Nunn, D., Simons, J. C., Gianatasio, C., Mueller, N., \& Eikermann, M. (2014). Anesthesia and increased hypercarbic drive impair the coordination between breathing and swallowing. 


\section{Figures}

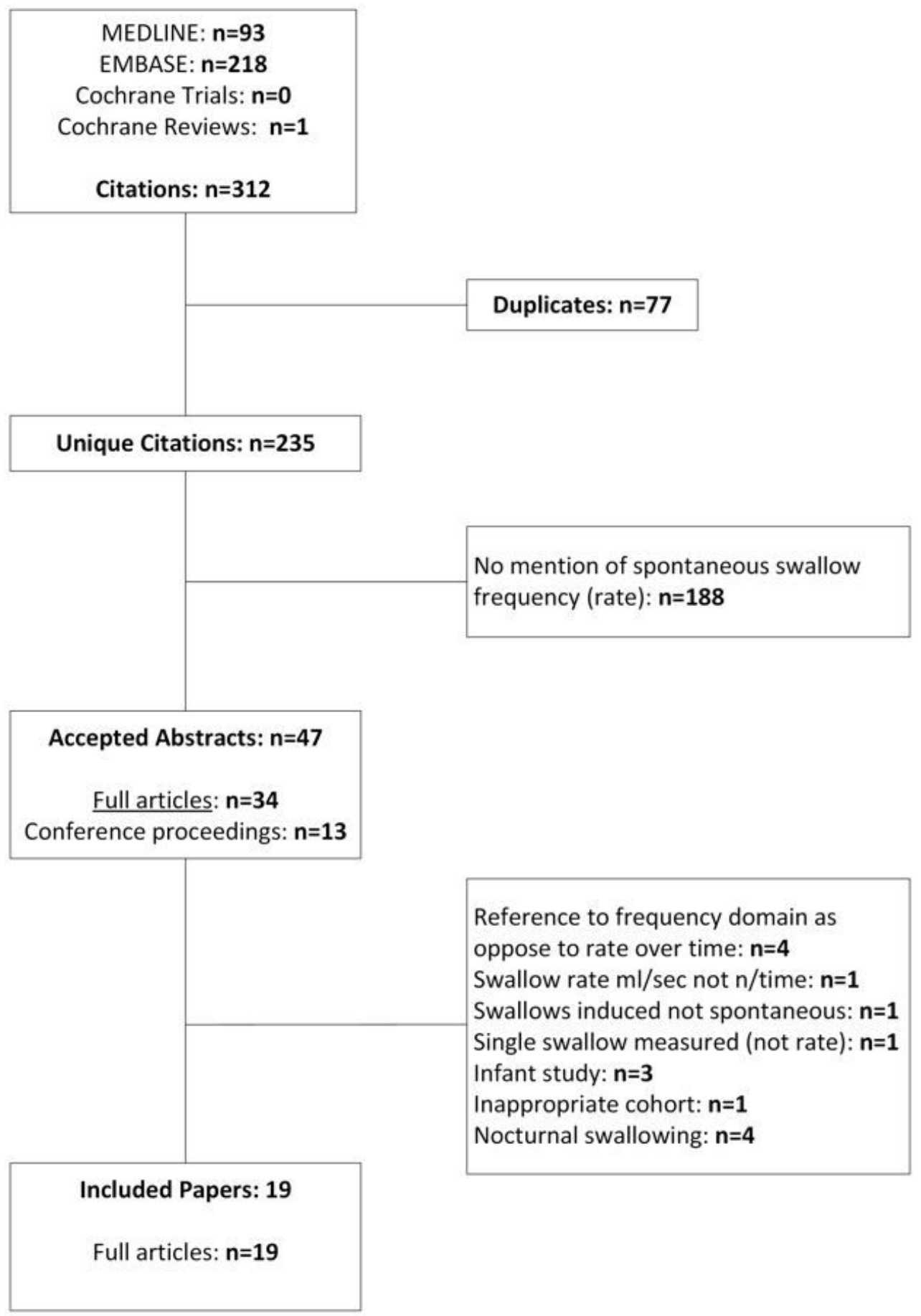

\section{Figure 1}

Flow chart illustrating the abstracts identified and reviewed. 


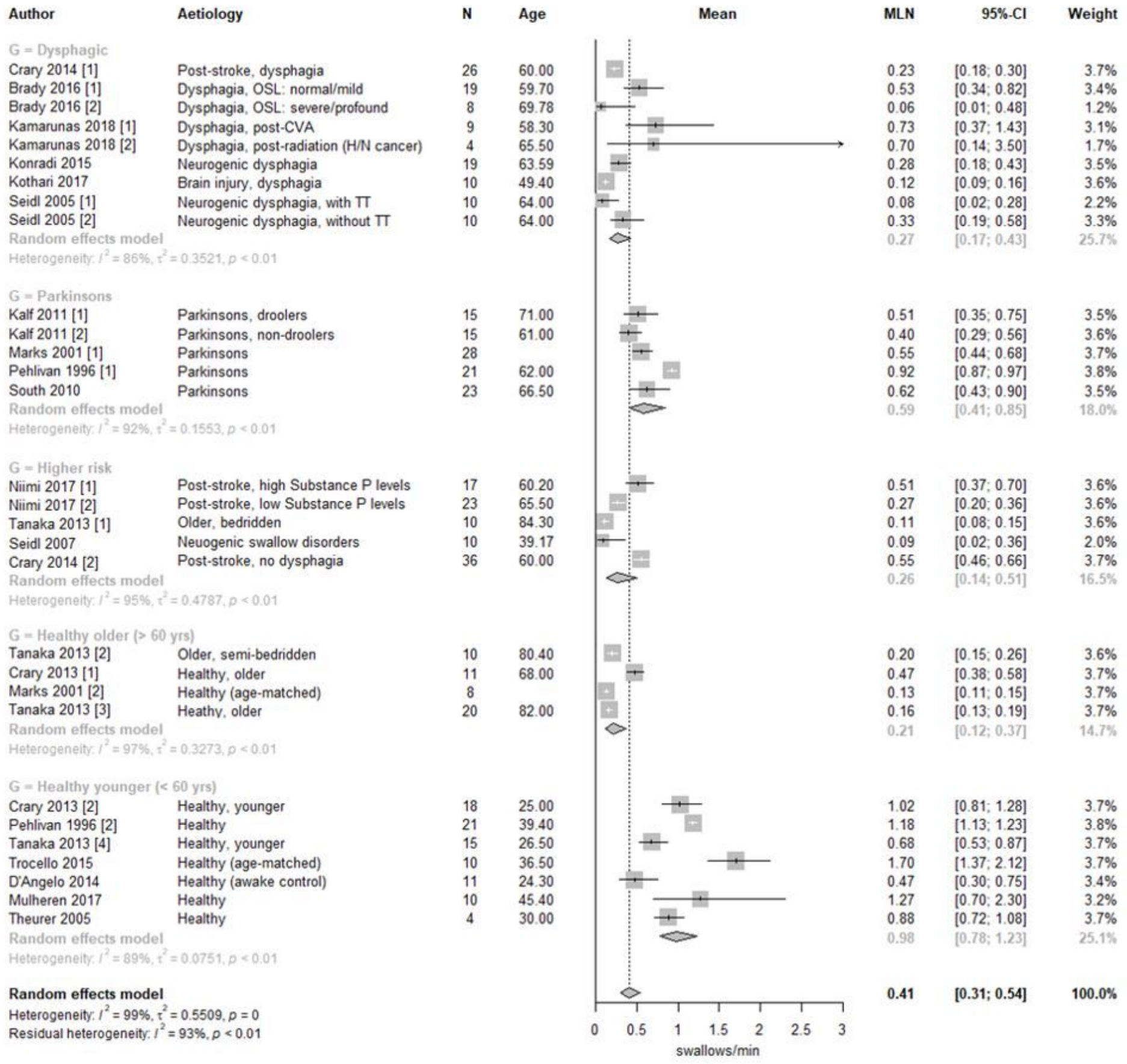

\section{Figure 2}

Forest plot of the means of study groups from papers categorised as having been diagnosed with dysphagia, Parkinson's disease, of being of higher risk of dysphagia, being healthy older ( $>60$ years) and healthy younger (< 60 years).

\section{Supplementary Files}

This is a list of supplementary files associated with this preprint. Click to download.

- Appendix.docx 
- PRISMA2009checklistv1.0.doc

Page 22/22 